\title{
Gamut Mapping through Perceptually-Based Contrast Reduction
}

\author{
Syed Waqas Zamir, Javier Vazquez-Corral, and Marcelo Bertalmío \\ Department of Information and Communication Technologies, Universitat Pompeu \\ Fabra, Barcelona, Spain \\ \{waqas.zamir, javier.vazquez, marcelo. bertalmio\}@upf .edu
}

\begin{abstract}
In this paper we present a spatial gamut mapping algorithm that relies on a perceptually-based variational framework. Our method adapts a well-known image energy functional whose minimization leads to image enhancement and contrast modification. We show how by varying the importance of the contrast term in the image functional we are able to perform gamut reduction. We propose an iterative scheme that allows our algorithm to successfully map the colors from the gamut of the original image to a given destination gamut while preserving the colors' perception and texture close to the original image. Both subjective and objective evaluation validate the promising results achieved via our proposed framework.
\end{abstract}

Keywords: Gamut Mapping (GM), Gamut Mapping Algorithm (GMA), color contrast, variational methods.

\section{Introduction}

The color gamut of a device is the set of colors that this device is able to reproduce. Different display systems have different color gamuts, making a process called gamut mapping (GM) essential: GM transforms colors from an input to an output gamut with the intention that a viewer watching the same image in different displays perceives the same colors. Intensive research has been carried out in the GM area; however, it is still an open field due to the difficulty of the challenges involved. One of the major tasks is to retain the perceived quality of the original image into the gamut mapped image and most of the algorithms in the literature lack in this regard.

A plethora of Gamut Mapping Algorithms (GMAs) exists in the literature and the interested reader is referred to the excellent book by Morovič [19]. In general, gamut mapping algorithms are classified into two broader categories. The first category consists of global (also called non-local or non-adaptive) GMAs [9], 11, 21, 22 that involve point-to-point mapping of colors (usually a predefined lookup table) from source to destination gamut. The standard non-local GM algorithm, Hue Preserving Minimum $\Delta E$ (HPMINDE), was proposed by Murch and Taylor 21] where, in order to reproduce the image, the out-of-gamut colors are clipped to closest points on the target gamut boundary along the lines of hue.

R. Klette, M. Rivera, and S. Satoh (Eds.): PSIVT 2013, LNCS 8333, pp. 1-11, 2014.

(C) Springer-Verlag Berlin Heidelberg 2014 
The non-adaptive GMAs involve either clipping or compression and completely ignore the spatial color configuration in the source image. In contrast, the second category involves the GMAs that take into account the spatial color information of the original image whilst fitting the color gamut of an image into the gamut of a given device [1, [2], 3], [12, [18, [20, 23. However, these algorithms are often computationally expensive, or based on many assumptions and may report halo artifacts. McCann [16, 17] proposed a Retinex-inspired framework that performs spatial comparisons to reproduce the image while preserving the local gradients at all scales as in the original image. A similar multi-resolution GM approach that adapts to the original image content is suggested by Farup et al. 10. A cluster based approach is defined in 14 for the optimization of GM. Alsam and Farup [2] proposed an iterative GMA that at iteration level zero gives the result identical to gamut clipping. However, by increasing the number of iterations, the solution approaches spatial gamut mapping. Unlike the global GMAs, the potential of spatial GM methods is to preserve the color gradient between two out-of-gamut colors instead of mapping them to the same in-gamut color. Another fundamental motivation behind spatial gamut mapping, in order to emulate the color perception properties of the Human Visual System (HVS), is the need to formulate a strategy where two out-of-gamut colors with identical lightness and chromaticity map to two different in-gamut colors depending on their spatial context in the image [12].

One of the major problems in GM is the evaluation of GMAs. Usually, subjective comparisons are performed [6], 8]. The most common subjective method is the pair comparison, where observers are asked to choose which of two different gamut-mapped versions of an image is more faithful to the original. However, subjective measures are time consuming, involve complexities, and do not provide clear cues to improve the given GMA. Recently, a perceptually-based color image difference metric [15] has been proposed that particularly emphasises on the assessment of gamut-mapped images. It is based on predicting the distortions in lightness, hue, chroma, contrast and structure of the gamut-mapped images by performing the comparison with the original images.

The contribution of this paper is to propose a perceptually inspired GMA where gamut reduction is achieved through contrast reduction, by adapting a framework [5, 4] that is inspired by the properties of contrast perception in the HVS and closely related to the Retinex theory of color vision [13. Our method outperforms state of the art techniques ([2], [14]) both subjectively and according to the aforementioned perceptually-based metric of [15].

This paper is organized as follows; first, the image energy functional presented in [5] for the perceptually inspired contrast enhancement is described in section 2. Then, in section 3, the contrast enhancement model is adapted to obtain our GM method. In section 4, experiments and results are discussed. Finally, the paper is concluded in section 5 . 


\section{Image Energy Functional}

Bertalmío et al. proposed an image enhancement model in [5], where the image energy functional is defined as

$$
\begin{aligned}
E(I)=\frac{\alpha}{2} \int_{\mathfrak{I}}\left(I(x)-\frac{1}{2}\right)^{2} d x & +\frac{\beta}{2} \int_{\mathfrak{I}}\left(I(x)-I_{0}(x)\right)^{2} d x \\
& -\frac{1}{2} \iint_{\mathfrak{I}^{2}} w(x, y)|I(x)-I(y)| d x d y
\end{aligned}
$$

where $\alpha \geq 0, \beta>0, I$ is a color channel $(R, G$ or $B), w(x, y)$ is a normalized Gaussian kernel of standard deviation $\sigma$, and $I(x)$ and $I(y)$ are two intensity levels at pixel locations $x$ and $y$ respectively.

This functional has two competing parts. The positive competing terms are global: the first one controls the dispersion from the middle gray value, which is assumed to be $1 / 2$ as described in the gray-world hypothesis [7], whereas the second term in the functional penalizes the departure from the original image $I_{0}$. The negative competing term represents the local contrast. Therefore, by minimizing the image energy $E(I)$, the aim is to maximize the contrast, while not departing too much from the original image, and to preserve the gray-world hypothesis. It is formulated in [5] that the steady state of the energy $E(I)$ can be achieved using the evolution equation

$$
I^{k+1}(x)=\frac{I^{k}(x)+\Delta t\left(\frac{\alpha}{2}+\beta I_{0}(x)+\frac{1}{2} R_{I^{k}}(x)\right)}{1+\Delta t(\alpha+\beta)}
$$

where the initial condition is $I^{k=0}(x)=I_{0}(x)$. The function $R_{I^{k}}(x)$ indicates the contrast function:

$$
R_{I^{k}}(x)=\frac{\sum_{y \in \mathfrak{I}} w(x, y) s_{m}\left(I^{k}(x)-I^{k}(y)\right)}{\sum_{y \in \mathfrak{I}} w(x, y)}
$$

where $x$ is a fixed image pixel and $y$ varies across the image. We define the slope function $s_{m}$ for a real constant $m>1$, when $d=I^{k}(x)-I^{k}(y)$, as follows

$$
s_{m}(d)= \begin{cases}-1, & \text { if }-1 \leq d \leq-\frac{1}{m} \\ m \cdot d, & \text { if }-\frac{1}{m}<d<\frac{1}{m} \\ +1, & \text { if } \frac{1}{m} \leq d \leq 1\end{cases}
$$

In [4] it is shown that the presented model is related to the Retinex theory of color vision proposed by Land 13 and that the model can be adapted to perform contrast reduction by just changing the sign of the contrast term. In the next section we will explain how we can use this contrast reduction property for gamut mapping. 


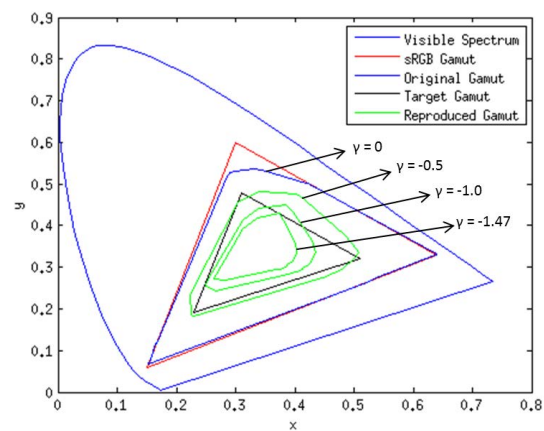

(a)

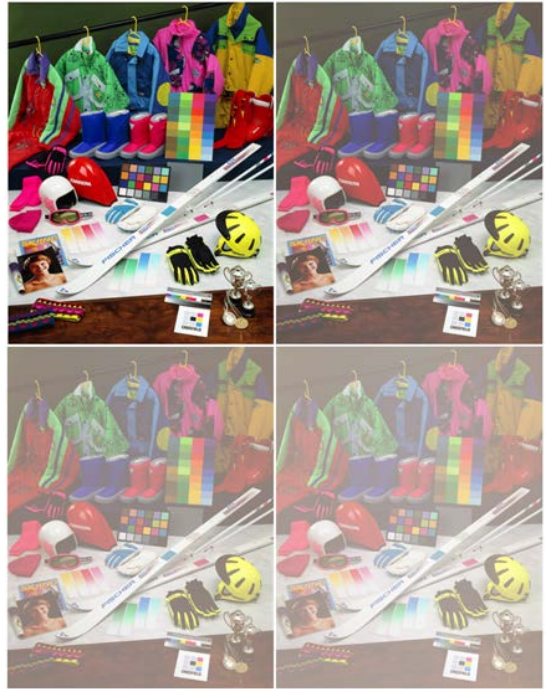

(b)

Fig. 1. Perceptual GM Approach. (a): Gamuts on chromaticity diagram. (b): Top left: original image. Top right: $\gamma=-0.50$. Bottom left: $\gamma=-1.0$. Bottom right: $\gamma=-1.47$.

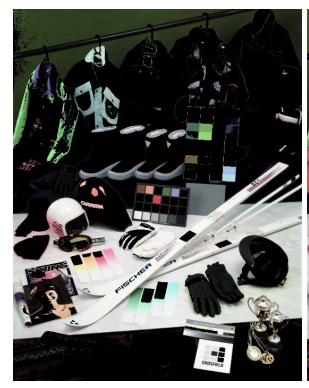

(a)

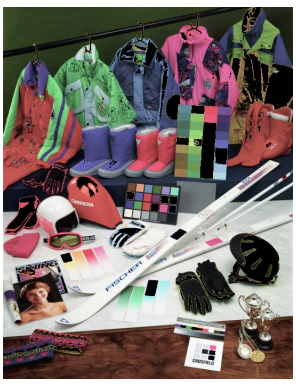

(b)

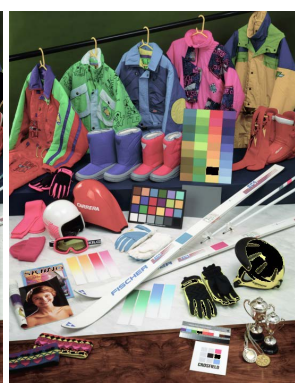

(c)

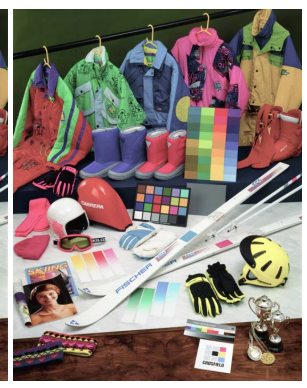

(d)

Fig. 2. Gradual mapping of colors. Out-of-gamut colors (in black) when (a): $\gamma=0$, (b): $\gamma=-0.50,(\mathrm{c}): \gamma=-1.0,(\mathrm{~d}): \gamma=-1.47$.

\section{Gamut Mapping Framework}

In this section, we adapt the image energy functional defined in Eq. (1) to perform gamut mapping from the gamut of the original image to the target gamut of a given device. In order to control the strength of the contrast modification, we add the contrast coefficient $\gamma$ in the image energy functional $E(I)$. Recall that $\alpha$ controls the dispersion around middle gray. Since, in the case of gamut 


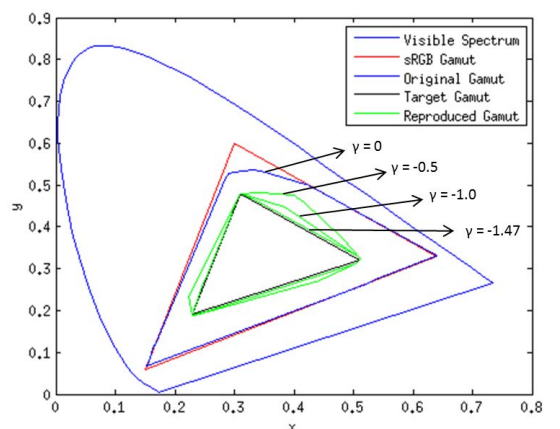

(a)

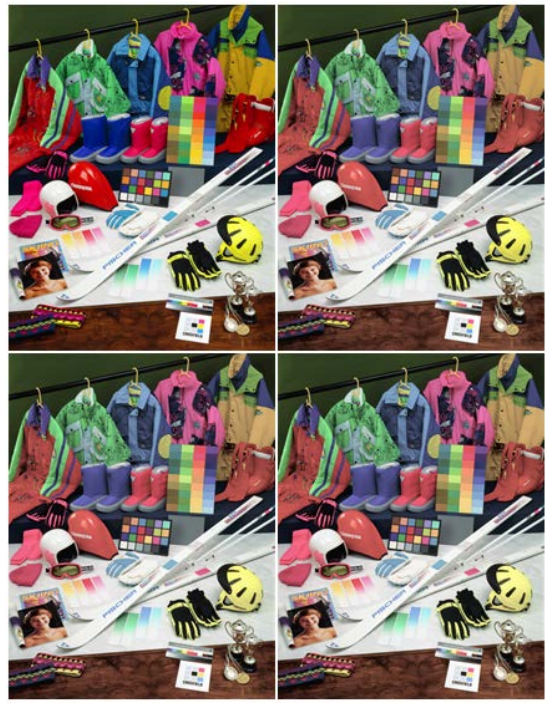

(b)

Fig. 3. Modified Perceptual GM Approach. (a): Gamuts on chromaticity diagram. (b): Top left: original image. Top right: $\gamma=-0.50$. Bottom left: $\gamma=-1.0$. Bottom right: $\gamma=-1.47$.

mapping, the HVS adapts to the luminance of the environment instead of the luminance of the stimulus, we set $\alpha=0$ and the image energy model defined in Eq. (11) becomes

$$
E(I)=\frac{\beta}{2} \int_{\mathfrak{I}}\left(I(x)-I_{0}(x)\right)^{2} d x-\frac{\gamma}{2} \iint_{\mathfrak{I}^{2}} w(x, y)|I(x)-I(y)| d x d y
$$

and subsequently the evolution equation (2) reduces to

$$
I^{k+1}(x)=\frac{I^{k}(x)+\Delta t\left(\beta I_{0}(x)+\frac{\gamma}{2} R_{I^{k}}(x)\right)}{1+\beta \Delta t}
$$

whereas, being $\gamma \in \mathbb{R}$ positive or negative depends on whether we want to maximize or minimize the contrast, respectively 4]. In this paper, $\gamma$ will always be negative, since our goal is to reduce the contrast in order to perform GM.

The evolution equation (6) has a steady state for each particular set of values for $\beta, \Delta t$ and $\gamma$. For example, in Fig. 1a, a chromaticity diagram is shown with different gamuts (visible spectrum, sRGB gamut, original gamut, target gamut and reproduced gamut). It can be seen that when $\gamma=0$ the steady state of the evolution equation is equivalent to the original image. In the same figure we show that as $\gamma$ decreases, the steady state of Eq. (6) has a gamut which is gradually smaller. Fig. 1a shows that, just by selecting an enough small gamma 


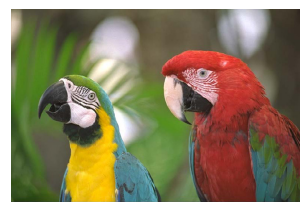

(a) original image

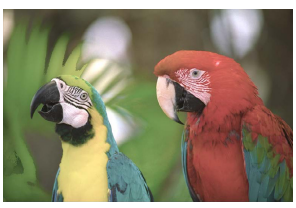

(b) $\sigma=25$

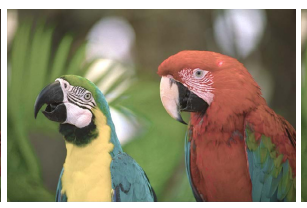

(c) $\sigma=100$

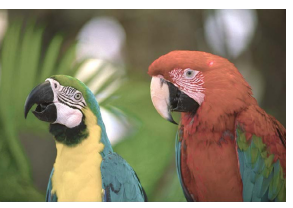

(d) $\sigma=200$

Fig. 4. Effect of standard deviation $(\sigma)$
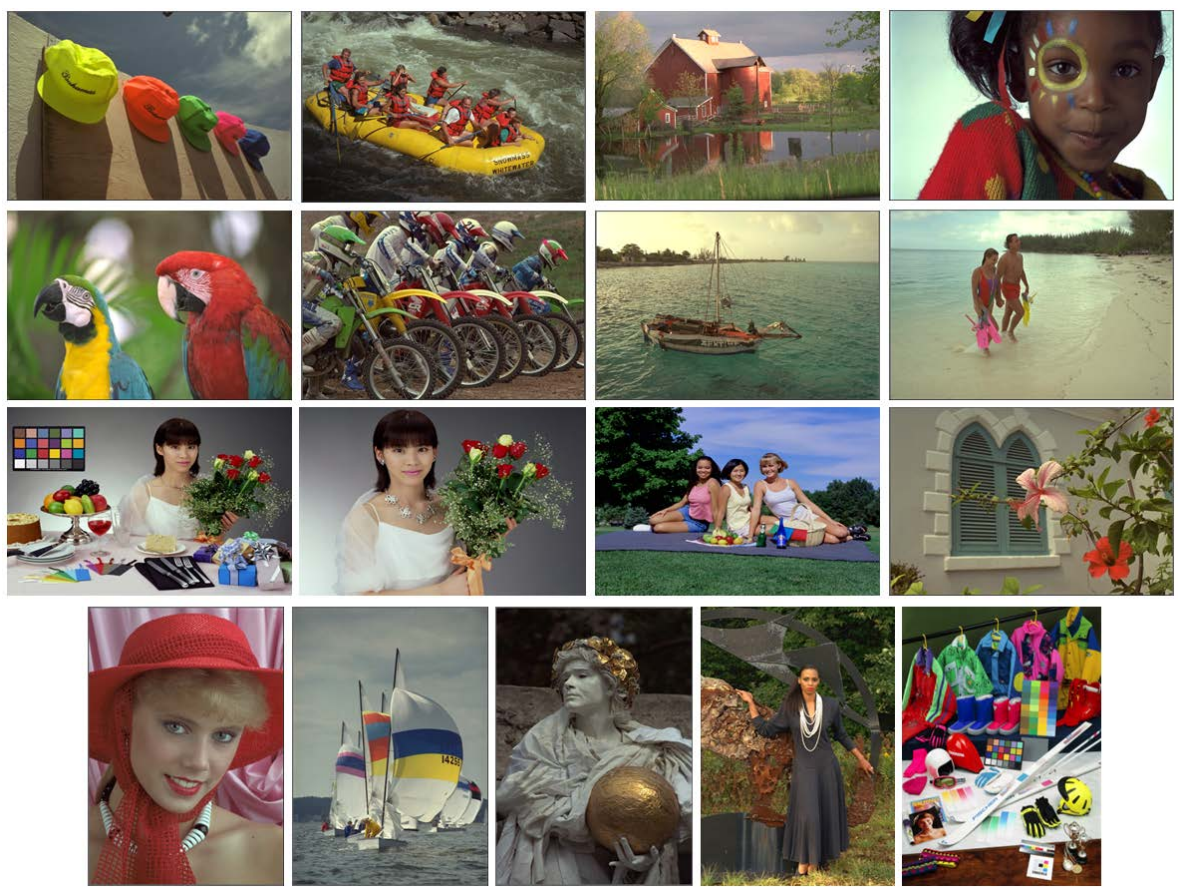

Fig. 5. Original sRGB images. Row 3; images 1, 2, 3, and Row 4; image 5 are from CIE 8 . Rest of the images are courtesy of Kodak.

$(\gamma=-1.47$ in this case) we are already performing a gamut mapping algorithm. However, in this case, colors that were originally inside the target gamut move inwards too much, and the appearance of the image becomes washed-up, as Fig. $1 \mathrm{~b}$ shows.

In order to improve the previous result, we present an iterative method in terms of the contrast coefficient $\gamma$. At iteration 1 , we set $\beta=1$ and $\gamma=0$, and therefore the original image is obtained as the steady state. We leave untouched the pixels that are inside the destination gamut, and we move to iteration 2 , where we decrease $\gamma$ (for example, setting $\gamma=-0.05$ ) and run Eq. (6) to steady 

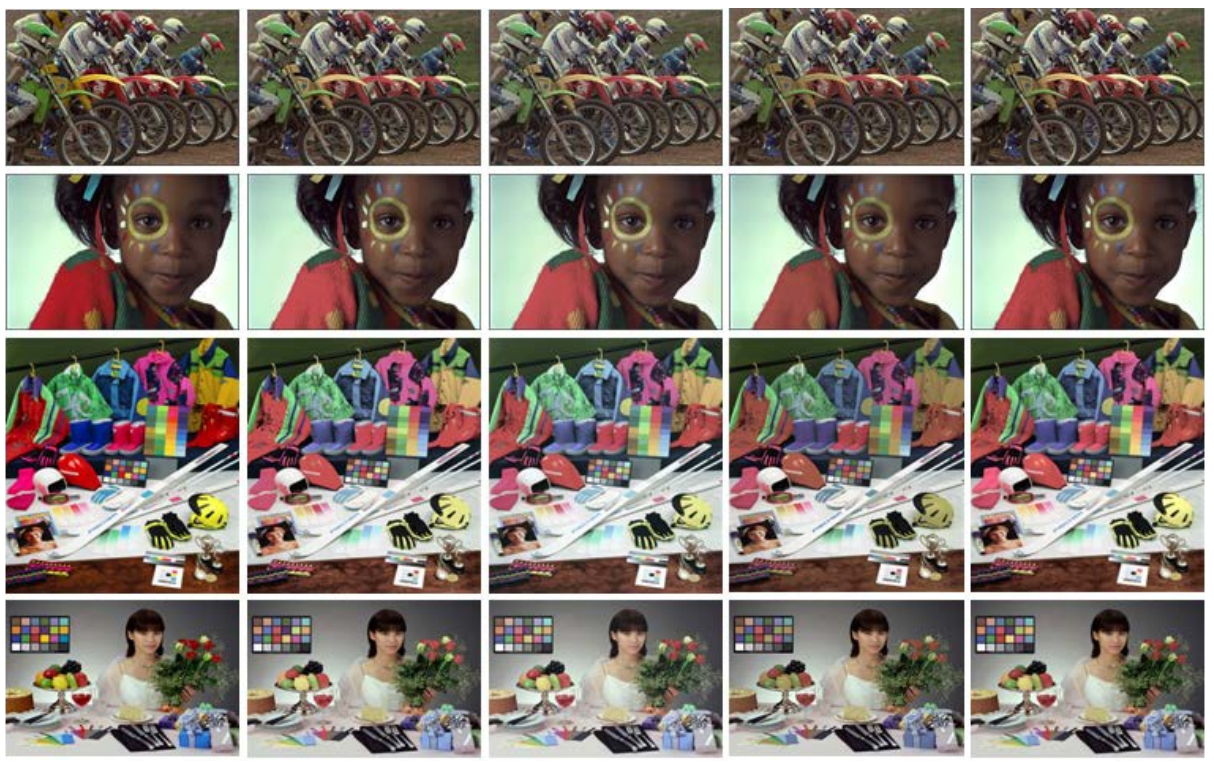

Fig. 6. Gamut mapping results. Column 1: original images. Columns 2: output of HPMINDE clipping [21]. Column 3: output of Lau et al. 14. Column 4: output of Alsam et al. 2]. Column 5: output of our algorithm.

state. In this second iteration, we check whether any of the points that were outside the gamut at the previous iteration have been moved inside the destination gamut. If this is the case, we leave them untouched for the following iterations. We keep iterating by decreasing $\gamma$ until all the out-of-gamut colors come inside the destination gamut. An example of this iterative procedure is shown in Fig. 2. where black pixels represent out-of-gamut pixels left in that iteration. It can be seen in Fig. 3a, that the reproduced gamut is covering a much wider range of colors than previously. It is shown in Fig. $3 \mathrm{~b}$ that the colors are better preserved as compared to the previous example (see Fig. 1b).

\section{Experiments}

We work in the RGB domain by fixing the parameters $\beta=1, \Delta t=0.10$ and iterate by decreasing the parameter $\gamma(\gamma \leq 0)$ until the colors of the original image come inside the target gamut. For each value of $\gamma$ we run Eq. (6) to steady state, which we assume that has been reached when the difference between two consecutive steps falls below $0.5 \%$. We have noticed that the standard deviation $\sigma$ of the Gaussian kernel $w$ is of great importance; we observe in Fig. 4 that a small value of $\sigma$ leads to the preservation of colors but introduces artifacts, whereas for the larger values of $\sigma$ each color pixel is strongly influenced from the surrounding colors. Therefore, we compute the gamut mapped images $\mathcal{I}_{\sigma}$ 


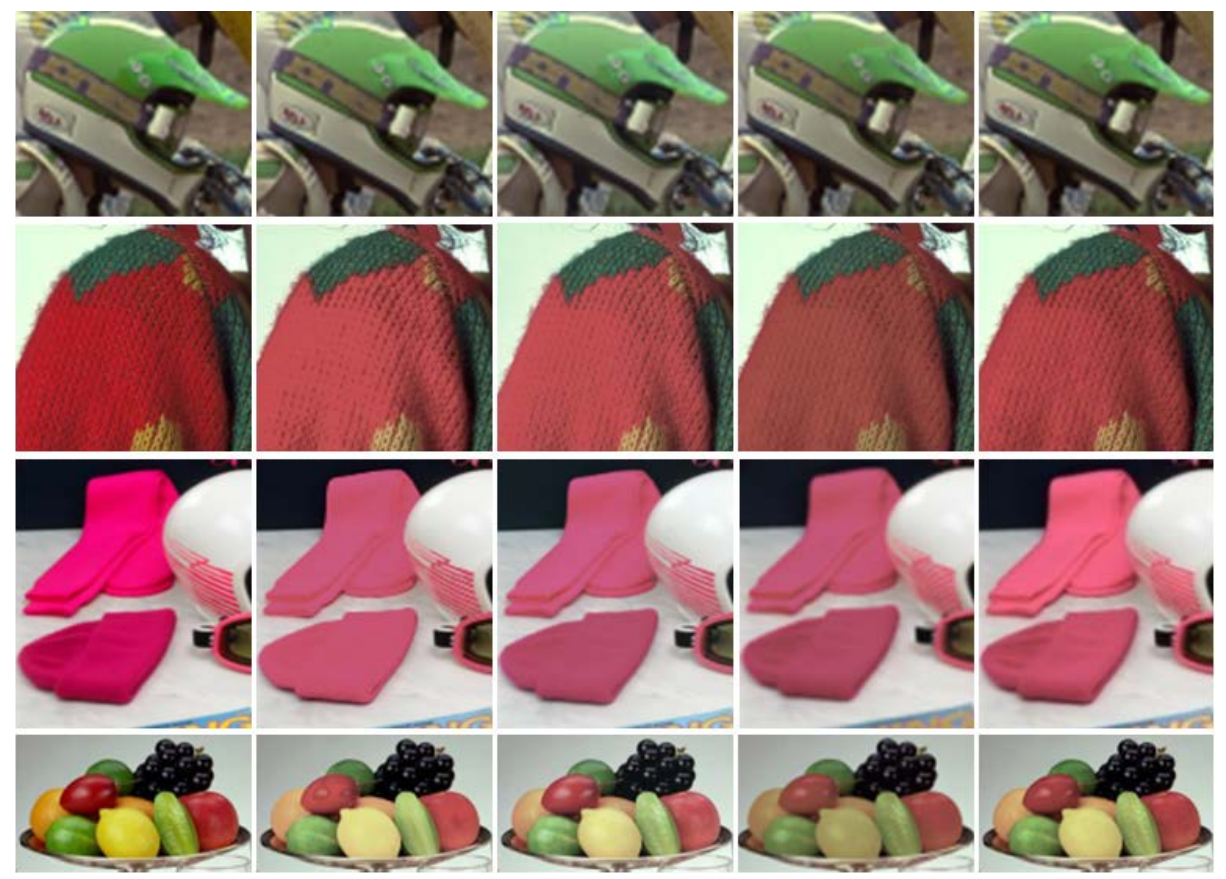

Fig. 7. Preserving details, all images are cropped from Fig. 6. Column 1: original cropped images. Column 2: output of HPMINDE [21. Column 3: output of Lau et al. 14]. Column 4: output of Alsam et al. 2]. Column 5: output of our algorithm.

by using four different values of standard deviations $\sigma \in\{50,100,150,200\}$. Subsequently, in order to obtain a final gamut mapped image $\mathcal{I}_{\text {final }}$, we combine all the outcomes $\mathcal{I}_{\sigma}$ with respect to the original image $\mathcal{I}_{\text {orig }}$, in Lab color space, by using the Delta-E measure.

$$
\mathcal{I}_{\text {final }}(x)=\underset{\mathcal{I}_{\sigma}}{\arg \min }\left(\operatorname{Lab}\left(\mathcal{I}_{\sigma}(x)\right)-\operatorname{Lab}\left(\mathcal{I}_{\text {orig }}(x)\right)\right)^{2}, \quad \sigma \in\{50,100,150,200\}
$$

We are confident that varying these parameters according to the application and image characteristics would give better results. However, our choice of parameters is the same for all the results shown in this paper.

\subsection{Qualitative Results}

In this section, we apply our method on a rather challenging target gamut as shown in Fig. 1a, The original sRGB images used are illustrated in Fig. 5. Given an image in sRGB, our algorithm maps the gamut of the original image into the destination gamut. The results presented in Fig. 6 show that our proposed 
Table 1. Quality assessment: perceptual difference measure 15]

\begin{tabular}{|l|c|c|c|c|}
\hline & $\begin{array}{c}\text { HPMINDE } \\
\text { Clipping [21 }\end{array}$ & Lau et al. [14] & Alsam et al. [2 & Our Algorithm \\
\hline Caps Image & 0.1027 & 0.1022 & 0.0821 & $\mathbf{0 . 0 7 1 1}$ \\
\hline Raft Image & 0.0772 & 0.0747 & 0.0857 & $\mathbf{0 . 0 4 7 1}$ \\
\hline Barn Image & 0.0268 & 0.0242 & 0.0134 & $\mathbf{0 . 0 0 8 8}$ \\
\hline Girl Image & 0.0825 & 0.0695 & 0.0359 & $\mathbf{0 . 0 2 0 9}$ \\
\hline Birds Image & 0.1829 & 0.1119 & $\mathbf{0 . 0 9 2 3}$ & 0.1086 \\
\hline Motorbikes Image & 0.0330 & 0.0396 & 0.0322 & $\mathbf{0 . 0 1 5 5}$ \\
\hline Boat Image & 0.0255 & 0.0187 & 0.0035 & $\mathbf{0 . 0 0 0 8}$ \\
\hline Beach Image & 0.0168 & 0.0151 & 0.0077 & $\mathbf{0 . 0 0 4 6}$ \\
\hline Party Image & 0.0569 & 0.0878 & 0.0487 & $\mathbf{0 . 0 2 8 0}$ \\
\hline Portrait Image & 0.0235 & 0.0393 & 0.0209 & $\mathbf{0 . 0 1 0 4}$ \\
\hline Picnic Image & 0.0954 & 0.0954 & $\mathbf{0 . 0 4 4 8}$ & 0.0638 \\
\hline Window Image & 0.0514 & 0.0591 & 0.0443 & $\mathbf{0 . 0 3 2 6}$ \\
\hline Woman with Hat Image & 0.1313 & 0.0882 & 0.0528 & $\mathbf{0 . 0 4 1 0}$ \\
\hline Sailing Boats Image & 0.0183 & 0.0287 & 0.0195 & $\mathbf{0 . 0 1 3 0}$ \\
\hline Statue Image & 0.0025 & 0.0061 & 0.0053 & $\mathbf{0 . 0 0 2 0}$ \\
\hline Model Image & 0.0292 & 0.0736 & 0.0398 & $\mathbf{0 . 0 3 9 0}$ \\
\hline Ski Image & 0.1899 & 0.1964 & 0.1734 & $\mathbf{0 . 1 0 4 0}$ \\
\hline
\end{tabular}

Table 2. Quality assessment: statistical data

\begin{tabular}{|l|c|c|c|}
\hline & Mean & Median & RMS \\
\hline HPMINDE Clipping [21] & 0.0674 & 0.0514 & 0.0873 \\
\hline Lau et al. [14] & 0.0665 & 0.0695 & 0.0807 \\
\hline Alsam et al. [2] & 0.0472 & 0.0398 & 0.0627 \\
\hline Our Algorithm & $\mathbf{0 . 0 3 6 0}$ & $\mathbf{0 . 0 2 8 0}$ & $\mathbf{0 . 0 4 8 5}$ \\
\hline
\end{tabular}

framework works well in preserving the colors, texture and color gradients from the out-of-gamut regions while staying faithful to the perception of the original image. For example, in Fig. 7 rows 1 and 4, it can be seen that the colors reproduced by our GM algorithm (fifth column) are much more saturated than those of HPMINDE 21] (second column), and the state of the art algorithms of Lau et al. [14] (third column) and Alsam et al. 2] (fourth column). Similarly, in Fig. 7 row 2, our algorithm not only reproduces the color efficiently but also preserves a great amount of texture. In Fig. 7. row 3, we can see our method accurately represents the difference in the lightness of identical hue (see the pink socks and pink beanie). Results show that our algorithm outperforms not only the widespread method [21] but also the state of the art algorithms [2], 14].

\subsection{Objective Quality Assessment}

Visually, the results presented so far underline the good performance of our GMA in terms of visual quality. This subjective outcome is backed by using the 
perceptual color quality measure presented in [15]: the Color Image Difference (CID) metric estimates the perceptual differences given by the changes, from one image to the other, in features such as hue, lightness, chroma, contrast and structure.

Comparisons using the CID metric are provided in Table 1 In this table we can see that our algorithm outperforms the other methods in 15 out of 17 test images. Moreover, the statistical data (mean, median and root mean square) is also presented in Table 2. These results show that our method produces a gamut mapped image which is, perceptually, more faithful to the original image as compared with the other methods.

\section{Conclusions}

In this paper, we have presented a gamut mapping algorithm based on a perceptually inspired variational framework. We have shown how to modify the variational framework in order to perform gamut mapping reduction. The main advantage of our method is its perceptual inspiration, that allows us to mimic some basic properties of the HVS while performing the mapping, and this is corroborated by the good scores we obtain with a perceptual metric for color and contrast distortion.

One subject left untreated in this paper is the need to develop a GMA that is capable of mapping colors from a smaller gamut to a larger gamut. In this direction, we are currently working on adapting our formulation to the problem of gamut extension.

Acknowledgement. This work was supported by the European Research Council, Starting Grant ref. 306337, and by Spanish grants ref. TIN2011-15954-E and ref. TIN2012-38112. The authors would like to thank Jan Morovič, Ivar Farup and Ali Alsam for their comments, suggestions and source codes. Many thanks go to Cheryl Lau for running experiments on a set of images.

\section{References}

1. Alsam, A., Farup, I.: Colour Gamut Mapping as a Constrained Variational Problem. In: Proc. 16th Scandinavian Conference on Image Analysis, pp. 109-118 (2009)

2. Alsam, A., Farup, I.: Spatial Colour Gamut Mapping by Orthogonal Projection of Gradients onto Constant Hue Lines. In: Proc. 8th International Symposium on Visual Computing, pp. 556-565 (2012)

3. Bala, R., Dequeiroz, R., Eschbach, R., Wu, W.: Gamut Mapping to Preserve Spatial Luminance Variations. Journal of Imaging Science and Technology, 122-128 (2001)

4. Bertalmío, M., Caselles, V., Provenzi, E.: Issues About Retinex Theory and Contrast Enhancement. International Journal of Computer Vision 83(1), 101-119 (2009)

5. Bertalmío, M., Caselles, V., Provenzi, E., Rizzi, A.: Perceptual Color Correction Through Variational Techniques. IEEE Transactions on Image Processing 16(4), 1058-1072 (2007) 
6. Bonnier, N., Schmitt, F., Brettel, H., Berche, S.: Evaluation of Spatial Gamut Mapping Algorithms. In: Proc. 14th Color Imaging Conference (2006)

7. Buchsbaum, G.: A Spatial Processor Model for Object Colour Perception. Journal of the Franklin Institute 310(1), 1-26 (1980)

8. CIE: Guidelines for the evaluation of gamut mapping algorithms. Technical Report (2004)

9. Ebner, F., Fairchild, M.D.: Gamut mapping from below: Finding minimum perceptual distances for colors outside the gamut volume. Color Research and Application, 402-413 (1997)

10. Farup, I., Gatta, C., Rizzi, A.: A multiscale framework for spatial gamut mapping. IEEE Transactions on Image Processing, 2423-2435 (2007)

11. Katoh, N., Ito, M., Ohno, S.: Three-dimensional gamut mapping using various color difference formulae and color spaces. Journal of Electronic Imaging 4(8), 365-379 (1999)

12. Kimmel, R., Shaked, D., Elad, M., Sobel, I.: Space-Dependent Color Gamut Mapping: A Variational Approach. IEEE Transactions on Image Processing, 796-803 (2005)

13. Land, E.H., McCann, J.J.: Lightness and Retinex Theory. Journal of the Optical Society of America, 1-11 (1971)

14. Lau, C., Heidrich, W., Mantiuk, R.: Cluster-based color space optimizations. In: Proc. IEEE International Conference on Computer Vision, pp. 1172-1179 (2011)

15. Lissner, I., Preiss, J., Urban, P., Lichtenauer, M.S., Zolliker, P.: Image-Difference Prediction: From Grayscale to Color. IEEE Transactions on Image Processing 22(2), 435-446 (2013)

16. McCann, J.J.: Lessons Learned from Mondrians Applied to Real Images and Color Gamuts. In: Proc. Color Imaging Conference, pp. 1-8 (1999)

17. McCann, J.J.: A Spatial Colour Gamut Calculation to Optimize Colour Appearance. Colour Image Science: Exploiting Digital Media, 213-233 (2002)

18. Meyer, J., Barth, B.: Color Gamut Matching for Hard Copy. In: Proc. SID Digest, pp. 86-89 (1989)

19. Morovič, J.: Color gamut mapping. John Wiley \& Sons (2008)

20. Morovič, J., Wang, Y.: A Multi-Resolution, Full-Colour Spatial Gamut Mapping Algorithm. In: Proc. Color Imaging Conference, pp. 282-287 (2003)

21. Murch, G.M., Taylor, J.M.: Color in Computer Graphics: Manipulating and Matching Color. In: Eurographics Seminar: Advances in Computer Graphics V, pp. 41-47 (1989)

22. Stone, M.C., Cowan, W.B., Beatty, J.C.: Color gamut mapping and the printing of digital color images. ACM Transactions on Graphics 7(4), 249-292 (1988)

23. Zolliker, P., Simon, K.: Retaining Local Image Information in Gamut Mapping Algorithms. IEEE Transactions on Image Processing 16(3), 664-672 (2007) 